\title{
Effectiveness and Safety of Favipiravir Compared to Hydroxychloroquine for Management of Covid-19: A Retrospective Study
}

\author{
Musim Alotaibi ${ }^{1,2}$ \\ Ahmed Ali ${ }^{1,3}$ \\ Duaa Bakhshwin ${ }^{4}$ \\ Yasser Alatawi ${ }^{5}$ \\ Sultan Alotaibi ${ }^{6}$ \\ Abdullah Alhifany (D) $^{7}$ \\ Badr Alharthi (D) ${ }^{2}$ \\ Nasser Alharthi ${ }^{2}$ \\ Awatef Alyazidi ${ }^{2}$ \\ Yasmeen Alharthi ${ }^{2}$ \\ Aziza Alrafiah (D) $^{8}$ \\ 'Department of Pharmacology, King \\ Abdulaziz University, Jeddah City, \\ Kingdom of Saudi Arabia; ${ }^{2}$ Department of \\ Pharmacy, King Faisal Medical Complex, \\ Taif City, Kingdom of Saudi Arabia; \\ ${ }^{3}$ Department of Pharmaceutics, Assiut \\ University, Assiut City, Egypt; \\ ${ }^{4}$ Department of Pharmacotherapy and \\ Outcomes Science, King Abdulaziz \\ University, Jeddah City, Kingdom of Saudi \\ Arabia; ${ }^{5}$ Department of Pharmacy \\ Practice, University of Tabuk, Tabuk City, \\ Kingdom of Saudi Arabia; ${ }^{6}$ Department of \\ Pharmacy, King Abdul-Aziz Medical, \\ Jeddah City, Kingdom of Saudi Arabia; \\ ${ }^{7}$ Department of Clinical Pharmacy \\ College of Pharmacy, Umm Al-Qura \\ University, Makkah, Kingdom of Saudi \\ Arabia; ${ }^{8}$ Department of Medical \\ Laboratory Technology, King Abdulaziz \\ University, Jeddah City, Kingdom of Saudi \\ Arabia
}

Background: Coronavirus disease (COVID-19) is an infectious disease due to SARS-COV -2. Patients with risk factors are vulnerable to severe morbidity and mortality. Favipiravir (FPV) and hydroxychloroquine (HCQ) are considered possible COVID-19 treatments.

Objective: To investigate the effectiveness and safety of FPV compared to HCQ in patients with COVID-19 as the standard of care approved by the national protocol there.

Methods: This is a retrospective cohort study on patients with COVID-19 who were administered either FPV or HCQ at King Faisal Medical Complex, Taif, Saudi Arabia, from June 2020 to August 2020.

Results: In total, 508 patients were included in the analysis. Patients were categorized into three groups by medication. Patients enrolled in this study were 244 (55.8\%) on FPV, $193(44.2 \%)$ on HCQ and 71 (13.81\%) on neither medication. Patients who received FPV had higher age and greater comorbidity. Most of the patients were discharged on day $14(\mathrm{n}=303,59.6 \%), 26(36.6 \%)$ in neither med, $154(63.1 \%)$ in FPV and $123(63.7 \%)$ in HCQ groups with significant difference between groups $(P<0.0001)$. Mortality rate was $8.2 \%(\mathrm{n}=20)$ in FPV and $7.3 \%(\mathrm{n}=14)$ in HCQ groups with significant difference between groups $(\mathrm{P}=0.048)$. Regarding drug safety, $19.7 \%$ of patients treated with FPV vs $7.8 \%$ HCQ have adverse effects with significant difference between groups $(P<0.0001)$. Most of the side effects were increase ALT and AST. Meanwhile, prolonged Q-T interval was reported only in the HCQ group (2.6\%). From Cox regression modeling, only mechanical ventilation due to Covid 19 was predictive for mortality (HR: 16.598, 95\% CI: 7.095$38.828, \mathrm{P}<0.0001)$. Meanwhile, there was no significant difference in the prediction of discharge of FPV (vs HCQ) (HR: $0.933,95 \%$ CI: $0.729-1.195, P=0.5843$ ), predictors of mortality were HCQ (vs FPV) (HR: 2.3, 95\% CI: 0.994-5.487, $P=0.0518$ ). Kaplan-Meier survival curves showed improved survival time and discharged time among patients in the HCQ versus FPV group with an insignificant difference between them $(P=0.85, P=0.06$, respectively).

Conclusion: The present study concluded that FPV and HCQ showed comparable efficacy in decrease mortality and oxygen requirements. FPV likely has a more favorable safety profile regarding cardiac toxicity. A randomized clinical trial with large patient numbers is recommended to confirm the effectiveness of these drugs in COVID-19 patients.

Keywords: coronavirus disease, COVID-19, favipiravir, hydroxychloroquine, mortality, risk factors

\section{Introduction}

P.O Box 80200, Jeddah, 21589, Kingdom of Saudi Arabia

Tel +966 I2640I000 Ext. 23495

Fax +966126401000 Ext. 21686

Email aalrafiah@kau.edu.sa
The World Health Organization approved coronavirus disease 2019 (COVID-19), a global pandemic on March 11th, 2019, resulting in millions of deaths. However, health authorities worldwide have officially authorized no therapy as a standard 
treatment agent for the virus. Therefore, health authorities around the world proposed several agents to be used in COVID-19 management. These agents include, for example, hydroxychloroquine (HCQ)/chloroquine, remdesivir, tocilizumab, prednisolone, and ivermectin. Unfortunately, many of these agents showed partial effectiveness but had many adverse effects. ${ }^{1-3}$ Furthermore, several of these medications were found to be at unacceptable levels in COVID-19 patients, particularly those with diabetes, hypertension, cardiovascular disease, renal failure, liver cirrhosis, stroke, and other co-morbidities. ${ }^{4}$ Furthermore, six additional Coronaviridae family members were known to produce infections in humans before discovering SARS-CoV-2, including the SARS-CoV infection, which was first reported in 2002, and the Middle East Respiratory Syndrome (MERS)-CoV infection, which was first reported in 2012. Because the genome sequence of SARS-CoV-2 shares about $75-80 \%$ similarity with SARS-CoV, and even more so with some bat coronaviruses, significant effort is being put into testing the efficacy of existing antiviral drugs for SARS-CoV -2 infection treatment, including those that are effective in other viral infections, such as ribavirin, lopinavir (ritonavir), and interferon. ${ }^{5}$ Hence, it is crucial to find safe and effective treatment options for this disease.

The utilization of chloroquine in COVID-19 patients became standard treatment ${ }^{6}$ after it was found to diminish infection in human cells ${ }^{7}$ and pose a favorable action on viral clearance and clinical outcome in a clinical trial. Following these findings, a group of regulatory experts and governments proved that chloroquine had antiCOVID-19 concrete action. The drug was included in the National Health Commission of the People's Republic of China's Republic guidelines for preventing, diagnosing, and therapy COVID-19-related pneumonia. ${ }^{8}$ One option to treat the novel COVID-19 virus is favipiravir (FPV), an RNA-dependent RNA polymerase (RdRp) inhibitor. It blocks the replication process by inhibiting the influenza virus's RdRp by adversely acting on genetic copying. It was approved by Japan Pharmaceuticals and Medical Devices Agency (PMDA) in 2014 as an antiviral for influenza. FPV has been included in COVID-19 treatment protocols after it showed promising results. ${ }^{9,10}$ However, few studies have been reported supporting FPV use for COVID-19 therapy. The Saudi Ministry of Health protocol for patients with confirmed COVID-19 added FPV for COVID-19 management. ${ }^{11}$

This study aimed to investigate the effectiveness and safety of FPV compared to hydroxychloroquine in patients with COVID-19 as the standard of care approved by the national protocol there.

\section{Materials and Methods Research Design and Population}

The present retrospective cohort study was implemented at King Faisal Medical Complex in Taif, Saudi Arabia, from June 2020 to August 2020. King Faisal Medical Complex provided access authorization to the hospital electronic medical records through Taif Research and Ethics Committee (Approval \# HAP-02-T-67) that was according to Declaration of Helsinki. Informed consent was waived as this was considered an exempt study. Health information was maintained in a confidential manner that prevents unauthorized use and disclosure to third parties. Included in this study were adult patients ( $\geq 18$ years) with a verified PCR diagnosis of COVID-19. Excluded from this study were pregnant, breastfeeding females, or patients $<18$ years.

According to $\mathrm{MOH}$ protocols, ${ }^{11}$ patients were classified into three groups: 1) FPV group: treated with FPV at a dose of $1800 \mathrm{mg} /$ dose twice daily on the first day then $800 \mathrm{mg} /$ dose two times daily for 7-10 days. 2) HCQ group: treated with HCQ at $400 \mathrm{mg} /$ dose twice daily on the first day, then $200 \mathrm{mg} /$ dose two times daily for 5-7 days. 3) and neither medications group.

\section{Baseline Information Collection}

Patients' information was gathered from medical records under the supervision of a competent medical professional. These data were demographic characteristics, comorbidities; vital signs; symptoms. As well as patient location (ward or intensive care unit [ICU]); type of respiratory support. The treatment impact on the outcomes was reported as mortality rate, discharge at day 14 , the adverse event of treatments.

\section{Statistical Analysis}

Data were analyzed utilizing SPSS software version 26 (IBM SPSS Statistics for Windows, version 26, IBM Corp., Armonk, N.Y., USA) and SAS (version 9.4) software. Kolmogorov-Smirnov test was used to check for the normality of data distributions. Continuous variables with normal distribution were presented as mean \pm (standard deviation [SD]); non-normal variables were reported as median (interquartile range $[\mathrm{IQR}]$ ). A comparison of parametric data was made using the Kruskal Wallis test. Categorical data were 
Table I Comparison of Demographic Characteristics of Patients According to Therapy Used

\begin{tabular}{|c|c|c|c|c|c|}
\hline Characteristics & $\begin{array}{l}\text { Total } \\
(n=508)\end{array}$ & $\begin{array}{l}\text { Neither Med } \\
(n=71)\end{array}$ & $\begin{array}{l}\text { Favipiravir } \\
(n=244)\end{array}$ & $\begin{array}{l}\text { Hydroxychloroquine } \\
(n=193)\end{array}$ & Significance \\
\hline $\begin{array}{l}\text { Age (years) } \\
\text { Mean } \pm \text { SD }\end{array}$ & $51.00 \pm 14.96(18.00-105.00)$ & $41.67 \pm 16.61(18.00-105.00)$ & $54.80 \pm 14.87(24.00-97.00)$ & $49.59 \pm 12.63(18.00-88.00)$ & $0.0001 *$ \\
\hline $\begin{array}{l}\text { Median (25-75 } \\
\text { IQR) }\end{array}$ & $51.00(40.00-61.00)$ & $38.00(28.00-51.00)$ & $54.00(45.00-65.00)$ & $49.00(41.00-57.00)$ & \\
\hline \multicolumn{5}{|l|}{ Gender } & \multirow[t]{3}{*}{0.284} \\
\hline Male & $306(60.2 \%)$ & $46(64.8 \%)$ & $152(62.3 \%)$ & $108(56.0 \%)$ & \\
\hline Female & $202(39.8 \%)$ & $25(35.2 \%)$ & $92(37.7 \%)$ & 85 (44.0\%) & \\
\hline \multicolumn{5}{|l|}{ Comorbidity } & \multirow[t]{3}{*}{$0.0001 *$} \\
\hline No & $226(44.5 \%)$ & $48(67.6 \%)$ & $82(33.6 \%)$ & $96(49.7 \%)$ & \\
\hline Yes & $282(55.5 \%)$ & $23(32.4 \%)$ & $162(66.4 \%)$ & $97(50.3 \%)$ & \\
\hline Diabetes mellitus & $167(32.9 \%)$ & $6(8.5 \%)$ & $105(43.0 \%)$ & $56(29.0 \%)$ & $0.0001 *$ \\
\hline Hypertension & $117(23.0 \%)$ & $13(18.3 \%)$ & $69(28.3 \%)$ & $35(18.1 \%)$ & 0.026 \\
\hline Heart diseases & $34(6.7 \%)$ & - & $26(10.7 \%)$ & $8(4.1 \%)$ & $0.00 I^{*}$ \\
\hline Asthma & $30(5.9 \%)$ & $\mathrm{I}(1.4 \%)$ & $14(5.7 \%)$ & $15(7.8 \%)$ & 0.149 \\
\hline Hypothyroidism & $27(5.3 \%)$ & $2(2.8 \%)$ & $17(7.0 \%)$ & $8(4.1 \%)$ & 0.256 \\
\hline Obese & $14(2.8 \%)$ & - & $10(4.1 \%)$ & $4(2.1 \%)$ & 0.136 \\
\hline
\end{tabular}

Notes: Data were expressed as mean \pm standard deviation (minimum-maximum) and median (25-75 IQR or number (\%) as appropriate. A comparison of parametric data was made using the Kruskal Wallis test. Comparison of categorized data was made using Pearson Chi-Square or Fisher Extract test as appropriate. *Significance at $<0.05$.

compared using the Person Chi-Square test and Fisher exact test as appropriate. Multivariable Cox regression models and Kaplan-Meier survival curves evaluated hospital stay duration and survival between groups. Statistical significance was defined as a $P$-value of less than 0.05 .

\section{Results}

\section{Comparison of Demographic Characteristics of Patients According to Therapy Used}

In this study, 508 COVID-19 patients were enrolled. Of them, 244 patients received FPV, 193 patients received HCQ, 71 patients did not receive either med. There was a significant difference between the mean of the age in different treatment groups $(P<0.0001$, Table 1$)$. The mean age was highest in the FPV group (54.80 \pm 14.87 years), then the HCQ group ( $49.59 \pm 12.63$ years) and least in neither med group ( $41.67 \pm 16.61$ years). In all the patients, age groups were mostly $\leq 65$ years $(n=431,84.8 \%)$ than $>65$ years $(\mathrm{n}=77,15.2 \%)$. There was a significant difference between age groups in different studied groups of patients (P $<0.0001)$. In neither med, FPV and HCQ groups, the proportion of males $(64.8 \%, 62.3 \%$ and $56.0 \%$, respectively) were higher than females $(35.2 \%, 37.7 \%$, and $44.0 \%$, respectively) with insignificant difference between groups $(P=0.284)$. In this study, $55.5 \%(\mathrm{n}=282)$ of patients have a history of diseases (comorbidities). There was a significant difference between groups $(P<0.0001)$ in terms of comorbidities. Comorbidities were highest in the FPV group (66.4\%), followed by the HCQ group (50.3\%), and least in the med group (32.4\%). In neither med, FPV and HCQ groups, the percentage of patients with diabetes mellitus were $(8.5 \%, 43.0 \%$ and $29.0 \% ; P<0.0001)$, hypertension $(18.3 \%, 28.3 \%$ and $18.1 \% ; P=0.026)$, asthma $(1.4 \%, 5.7 \%$ and $7.8 \% ; P=0.149)$, hypothyroidism $(2.8 \%, 7.0 \%$ and $4.1 \% ; P=0.256$ ). Meanwhile, patients in FPV and HCQ groups only had heart diseases $(10.7 \%$ and $4.1 \%$; $P=0.001)$ and obesity $(4.1 \%$ and $2.1 \% ; P=0.136)$ (Table 1$)$.

\section{Comparison of Clinical Characteristics of Patients According to Therapy}

Table 2 shows a comparison of clinical characteristics of patients between groups. In all groups, the manifestations of COVID-19 were mostly cough $(83.7 \%)$, then fever $(69.9 \%)$, low oxygen saturation (66.1\%), shorting of breath $(54.3 \%)$, headache $(23.6 \%)$, running nose $(4.3 \%)$, diarrhea (2.6\%) and vomiting (1.7\%). In neither med, FPV 
Table 2 Comparison of Clinical Characteristics of Patients According to Therapy

\begin{tabular}{|c|c|c|c|c|c|}
\hline Characteristics & $\begin{array}{l}\text { Total } \\
(n=508)\end{array}$ & $\begin{array}{l}\text { Neither Med } \\
(n=7 I)\end{array}$ & $\begin{array}{l}\text { Favipiravir } \\
(n=244)\end{array}$ & $\begin{array}{l}\text { Hydroxychloroquine } \\
(n=193)\end{array}$ & Significance \\
\hline \multicolumn{6}{|l|}{ Symptoms (n, \%) } \\
\hline Cough & 425 (83.7\%) & 49 (69.0\%) & $20 \mathrm{I}(82.4 \%)$ & I 75 (90.7\%) & $0.000 I^{*}$ \\
\hline Fever & $355(69.9 \%)$ & $33(46.5 \%)$ & 177 (72.5\%) & 145 (75.1\%) & $0.000 I^{*}$ \\
\hline $\begin{array}{l}\text { Low oxygen } \\
\text { saturation }\end{array}$ & $336(66.1 \%)$ & 9 (12.7\%) & $176(72.1 \%)$ & I5I (78.2\%) & $0.000 I^{*}$ \\
\hline Shorting of breath & $276(54.3 \%)$ & $5(7.0 \%)$ & 146 (59.8\%) & $125(64.8 \%)$ & $0.000 I^{*}$ \\
\hline Headache & $120(23.6 \%)$ & 60 (84.5\%) & $36(14.8 \%)$ & $24(12.4 \%)$ & $0.000 I^{*}$ \\
\hline Running nose & $22(4.3 \%)$ & 21 (29.6\%) & - & I (0.5\%) & $0.000 I^{*}$ \\
\hline Diarrhea & $13(2.6 \%)$ & $3(4.2 \%)$ & $9(3.7 \%)$ & I (0.5\%) & 0.072 \\
\hline Vomiting & 7 (I.4\%) & $6(8.5 \%)$ & I (0.4\%) & - & $0.000 I^{*}$ \\
\hline \multicolumn{5}{|c|}{ Respiratory support (n, \%) } & \multirow[t]{4}{*}{$0.0001 *$} \\
\hline No oxygen & $112(22.0 \%)$ & 34 (47.9\%) & $44(18.0 \%)$ & $34(17.6 \%)$ & \\
\hline Oxygen only & $370(72.8 \%)$ & $37(52.1 \%$ & 179 (73.4\%) & 154 (79.8\%) & \\
\hline Mechanical ventilation & $26(5.1 \%)$ & - & $21(8.6 \%)$ & $5(2.6 \%)$ & \\
\hline \multicolumn{5}{|l|}{ Patient location (n, \%) } & \multirow[t]{3}{*}{$0.002^{*}$} \\
\hline Ward & 462 (90.9\%) & 70 (98.6\%) & $211(86.5 \%)$ & I8I (93.8\%) & \\
\hline$I C U$ & 46 (9.1\%) & I (I.4\%) & 33 (13.5\%) & $12(6.2 \%)$ & \\
\hline
\end{tabular}

Notes: Data were expressed as number (\%) as appropriate. Comparison of categorized data was made using Pearson Chi-Square or Fisher Extract test as appropriate. *Significance at $<0.05$.

and HCQ groups, the percentage of patients with cough were $(69.0 \%, 82.4 \%$ and $90.7 \%, P<0.0001)$, fever (46.5\%, 72.5\% and 75.1\%, $P<0.0001)$, low oxygen saturation $(12.7 \%, 72.1 \%$ and $78.2 \%, P<0.0001)$, shorting of breath $(7.0 \%, 59.8 \%$ and $64.8 \%, P<0.0001)$, headache $(84.5 \%, 14.8 \%$ and $12.4 \%, P<0.0001)$ and diarrhea $(4.2 \%$, $3.7 \%$ and $0.5 \%, P=0.072$ ). Meanwhile, in neither med and HCQ groups only running nose was observed $(29.6 \%$ and $0.5 \%, P<0.0001$ ), and in neither med and FPV groups, only vomiting was observed $(8.5 \%$ and $0.4 \%, P<0.0001)$.

Regarding respiratory support, most patients were on oxygen only $(72.8 \%)$, then on no oxygen $(22.0 \%)$ and least was on mechanical ventilation $(5.1 \%)$. The percentage of patients in neither med, FPV, and HCQ groups on no oxygen were $(47.9 \%, 18.0 \%$, and $17.6 \%$, respectively). Those who received oxygen only $(52.1 \%, 73.4 \%$, and $79.8 \%$, respectively) and those on mechanical ventilation were in FPV and HCQ groups only $(9.5 \%$ and $2.8 \%$, respectively) with significant differences between groups $(P<0.0001)$. Most of the patients were in Ward $(90.9 \%, \mathrm{n}=$ 462), while only $9.1 \%(n=46)$ were admitted to ICU. The patients in Ward and ICU in neither med group were (98.6\% and 1.4\%), FPV treated group $(86.5 \%$ and $13.5 \%$ ), and in HCQ treated group (93.8\% and $6.2 \%)$ with significant difference between groups $(P=0.002)$, (Table 2).

\section{Comparison of Clinical Outcomes According to Therapy}

Table 3 shows the outcomes of patients in different treatment groups. Most of the patients discharge at the end of treatment $(n=303,59.6 \%), 26(36.6 \%)$ in neither med, 154 $(63.1 \%)$ in FPV and $123(63.7 \%)$ in HCQ groups with significant differences between groups $(P<0.0001)$. The number of patients transfer to ICU were $4(5.8 \%)$ in neither med, $9(4.3 \%)$ in FPV and $11(6.0 \%)$ in HCQ groups with no significant difference between groups $(P=0.715)$. There was a significant difference in mortality between groups $(\mathrm{P}=0.048)$. Mortality rate was $8.2 \%$ $(n=20)$ in FPV and 7.3\% $(n=14)$ in HCQ groups. (Table 3).

\section{Multivariable Cox Regression Model for Mortality and Discharge Prediction}

In the multivariable Cox regression model 1 of mortality prediction utilizing the patient group-administered FPV as a reference, therapy with HCQ (vs FPV) (HR: 2.3,95\% CI: 0.994-5.487, $\mathrm{P}=0.0518$ ) not find statistical significance. Only mechanical ventilation due to Covid 19 was 
Table 3 Comparison of Clinical Outcomes According to Therapy

\begin{tabular}{|c|c|c|c|c|c|}
\hline Characteristics & Total $(n=508)$ & Neither Med $(n=7 I)$ & Favipiravir $(n=244)$ & Hydroxychloroquine $(n=193)$ & Significance \\
\hline \multicolumn{5}{|c|}{ Discharge at day 14} & \multirow[t]{3}{*}{$0.000 I^{*}$} \\
\hline Yes & 303 (59.6\%) & $26(36.6 \%)$ & $154(63.1 \%)$ & $123(63.7 \%)$ & \\
\hline No & 205 (40.4\%) & 45 (63.4\%) & 90 (36.9\%) & $70(36.3 \%)$ & \\
\hline \multicolumn{5}{|c|}{ Transfer to ICU } & \multirow[t]{3}{*}{0.715} \\
\hline Yes & $24(5.2 \%)$ & $4(5.8 \%)$ & $9(4.3 \%)$ & II (6.0\%) & \\
\hline No & 439 (94.8\%) & 65 (94.2\%) & $202(95.7 \%)$ & $172(94.0 \%)$ & \\
\hline \multicolumn{5}{|c|}{ Death } & \multirow[t]{3}{*}{$0.048 *$} \\
\hline Yes & $34(6.7 \%)$ & - & $20(8.2 \%)$ & 14 (7.3\%) & \\
\hline No & 474 (93.3\%) & 71 (100.0\%) & 224 (91.8\%) & 179 (92.7\%) & \\
\hline
\end{tabular}

Notes: Data were expressed as number (\%) as appropriate. Comparison of categorized data was made using Pearson Chi-Square or Fisher Extract test as appropriate. *Significance at $<0.05$.

predictive for mortality (HR: 16.598, 95\% CI: 7.095$38.828, \mathrm{P}<0.0001)$. While other factors were not predictor for mortality in that model as age $>65$ years (HR: 0.898 , 95\% CI: 0.321-2.517, $\mathrm{P}=0.8386$ ), female gender (HR: $0.762,95 \%$ CI: $0.310-1.870, \mathrm{P}=0.5523)$ and comorbidity (HR: $2.159,95 \%$ CI: 0.834-5.592, $\mathrm{P}=0.1129$ ).

Multivariable for discharge prediction in model 2 revealed significantly high risk of extended hospitalization were neither med versus HCQ (HR: $0.540,95 \% \mathrm{CI}$ : $0.350-0.834, P=0.0054$ ), age $>65$ years (HR: 0.630 , 95\% CI: $0.438-0.906, P=0.0126)$ and mechanical ventilation (HR: $0.095,95 \%$ CI: $0.023-0.380, P=0.0009$ ). In contrast, there was no significant difference in prediction of discharge of FPV (vs HCQ) (HR: 0.933, 95\% CI:
$0.729-1.195, P=0.5843$ ), female gender (HR: 1.182, 95\% CI: $0.933-1.497, P=0.1670$ ) and comorbidity (HR: 0.878, 95\% CI: $0.689-1.118, P=0.2908$ ) (Table 4).

\section{Comparison of Adverse Effects Between FPV and HCQ}

The drugs' adverse effects were significantly higher in FPV versus HCQ groups $(19.7 \%$ versus $7.8 \%$, $P<0.0001)$. There was a significant increase in FPV versus HCQ groups in ALT level $(13.9 \%$ and $2.6 \%$, $P<0.0001)$ and AST level $(8.6 \%$ and $1.0 \%, P<0.0001)$. Meanwhile, there were no significant difference in FPV versus HCQ groups in vomiting $(2.9 \%$ and $2.1 \%$, $P=0.418)$, nausea $(1.6 \%$ and $1.6 \%, P=0.627)$, diarrhea

Table 4 Multivariable Cox Regression Model for Mortality and Discharge Prediction

\begin{tabular}{|l|l|l|l|}
\hline Parameters & P-value & Hazard Ratio & 95\% Hazard Ratio Confidence Limits \\
\hline Model I: Multivariable for mortality prediction. \\
\hline Hydroxychloroquine (vs Favipiravir) & 0.0518 & 2.335 & $0.994-5.487$ \\
Age > 65 years & 0.8386 & 0.898 & $0.321-2.517$ \\
Female gender & 0.5523 & 0.762 & $0.310-1.870$ \\
Mechanical ventilation & $<0.000 I^{*}$ & 16.598 & $7.095-38.828$ \\
Comorbidity & 0.1129 & 2.159 & $0.834-5.592$ \\
\hline Model 2: Multivariable for discharge prediction. & \multicolumn{3}{|l}{} \\
\hline Favipiravir (vs Hydroxychloroquine) & 0.5843 & 0.933 & $0.729-1.195$ \\
Neither Med (vs Hydroxychloroquine) & $0.0054^{*}$ & 0.540 & $0.350-0.834$ \\
Age > 65 years & $0.0126^{*}$ & 0.630 & $0.438-0.906$ \\
Female gender & 0.1670 & 1.182 & $0.933-1.497$ \\
Mechanical ventilation & $0.0009^{*}$ & 0.094 & $0.023-0.380$ \\
Comorbidity & 0.2908 & 0.878 & $0.689-1.118$ \\
\hline
\end{tabular}

Note: *Significance at $<0.05$. 
Table 5 Comparison of Adverse Effects Between Favipiravir and Hydroxychloroquine

\begin{tabular}{|l|l|l|l|}
\hline Adverse Effects & Favipiravir (n=244) & Hydroxychloroquine (n=193) & Significance \\
\hline No & $196(80.3 \%)$ & $178(92.2 \%)$ & $0.000 I^{*}$ \\
\hline Yes & $48(19.7 \%)$ & $15(7.8 \%)$ & $5(2.6 \%)$ \\
\hline Increased ALT & $34(13.9 \%)$ & $2(1.0 \%)$ & $0.000 I^{*}$ \\
\hline Increased AST & $21(8.6 \%)$ & $4(2.1 \%)$ & $0.000 I^{*}$ \\
\hline Vomiting & $7(2.9 \%)$ & $3(1.6 \%)$ & 0.418 \\
\hline Nausea & $4(1.6 \%)$ & $2(1.0 \%)$ & 0.627 \\
\hline Diarrhea & $4(1.6 \%)$ & $5(2.6 \%)$ & 0.458 \\
\hline Prolonged Q-T interval & - & & $0.016 *$ \\
\hline Patients stop medications because of adverse effects & $4(1.8 \%)$ & $4(2.1 \%)$ & $189(97.9 \%)$ \\
\hline Yes & $240(98.4 \%)$ & & 0.504 \\
\hline No & & \\
\hline
\end{tabular}

Notes: Data were expressed as numbers (\%). Comparison of categorized data was made using Pearson Chi-Square or Fisher Extract test as appropriate. *Significance at $<0.05$.

(1.6\% and $1.0 \%, P=0.458)$. Prolonged Q-T interval was reported only in the HCQ group (2.6\%). Patients stop the medication due to adverse effects were $4(1.8 \%)$ in FPV and $4(2.1 \%)$ in HCQ groups with no significant difference between groups $(\mathrm{P}=0.504)$ (Table 5).

\section{Kaplan-Meier Survival Curves Among All Patient Treatment Groups}

Kaplan-Meier survival curves revealed improved survival between patients in HCQ therapy versus the FPV group (Figure 1). However, the mean survival time and standard error for the FPV group were $26.40 \pm 0.43$ days, and for the HCQ group was $27.06 \pm 0.60$ days with a non-significant difference between them $(\mathrm{P}=0.8554)$. Kaplan-Meier survival curves showed improved discharge time among patients in the HCQ versus FPV group (Figure 2). However, the mean discharge time and standard error for the FPV group was 16.64 \pm 0.63 days, and for the HCQ group was $14.051 \pm 0.63$ days, with an insignificant difference between them $(\mathrm{P}=0.060)$.

\section{Discussion}

The need for safe and efficient treatment options continues to be the main point of clinical research despite the advent of COVID-19 vaccines. Before the pandemic, scientists postulated that HCQ interacted with the virus, and in vitro evidence indicated an antiviral effect. ${ }^{9,12}$

FPV is an RNA-dependent RNA polymerase inhibitor promoted in China and Japan to treat influenza outbreaks. ${ }^{13}$ Therefore, there was a considerable need for diverse research testing its efficacy and safety in such a setting, based on the limited published evidence about its usage in COVID-19 treatment. So that, the current retrospective cohort research aimed to study FPV's effectiveness and safety in managing COVID-19 cases in Saudi Arabia.

The first clinical study of FPV was non-randomized interventional research consisting of 80 cases with non-severe COVID-19 compared FPV to lopinavir/ritonavir and found that the FPV group improved viral clearance on day 7, indicating that FPV could be useful in the treatment of COVID-19. At day 14, FPV patients showed improved chest CT scans 32/ 35 (91.43\%) versus lopinavir/ritonavir patients 28/45 $(62.22 \%)(p=0.004) .{ }^{10}$ Moreover, Rattanaumpawan et $\mathrm{al}^{14}$ reported retrospective research included 274 COVID-19 cases admitted to 5 Thai hospitals, of whom 63 (23.0\%) got FPV. About all cases were prescribed HCQ-based drug (98.4\%) and a protease inhibitor (96.8\%) within two days of starting FPV treatment; half of the cases were given azithromycin (49.2\%). The research showed that FPV is successful in treating COVID-19 patients.

In demographic data, most of the patients were men in the FPV group (60.2\%) and HCQ group (62.3\%), with insignificant differences between groups. Above $60 \%$ of men also showed in another study. ${ }^{15}$ FPV is mainly received by the middle-aged group, like in other studies. ${ }^{15,16}$ Comorbidities like hypertension, diabetes mellitus, and heart disease were manifested in both groups (FPV group and HCQ group). Researchers also found 


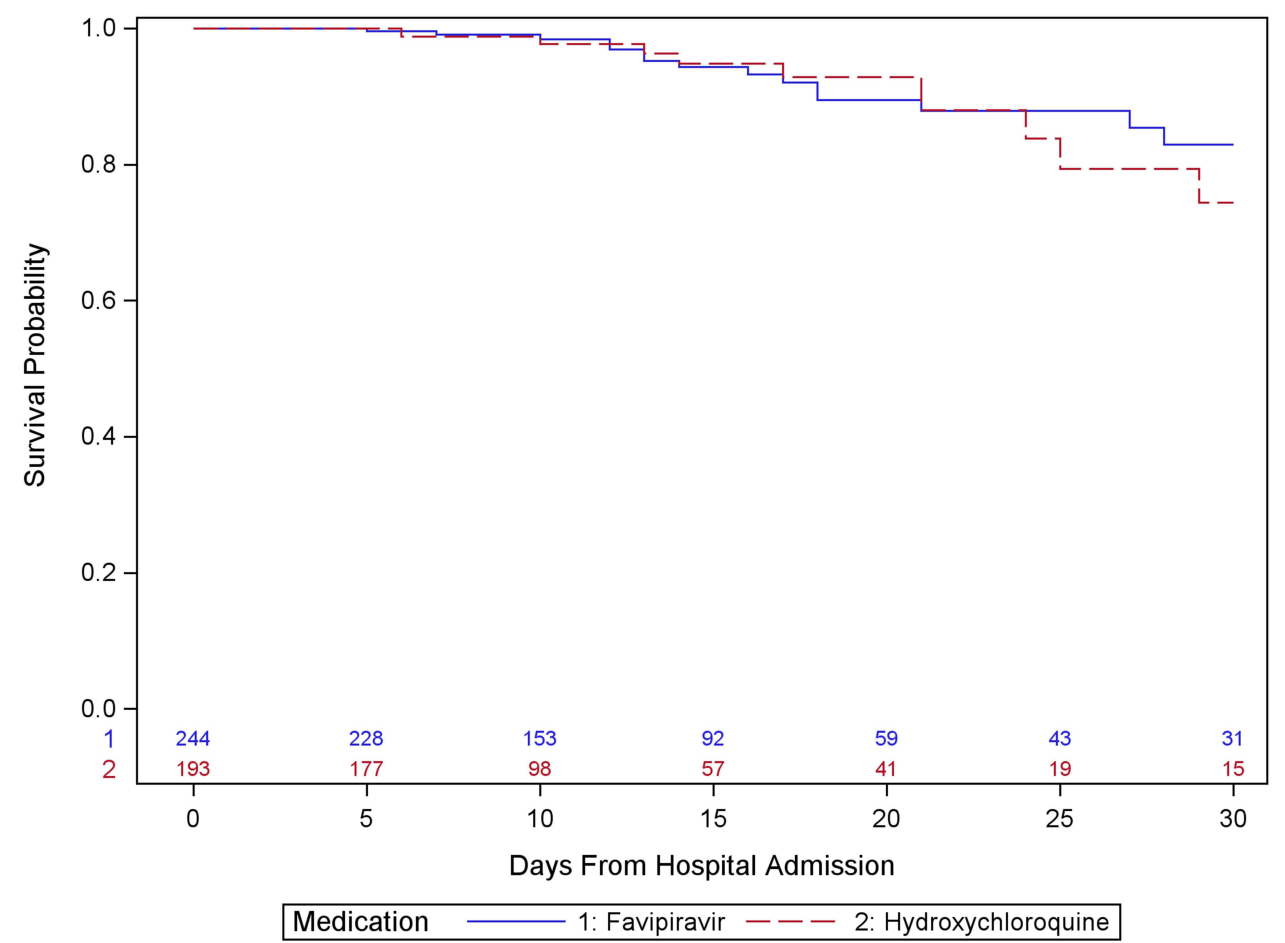

Figure I Kaplan-Meier survival curves among treatment two groups.

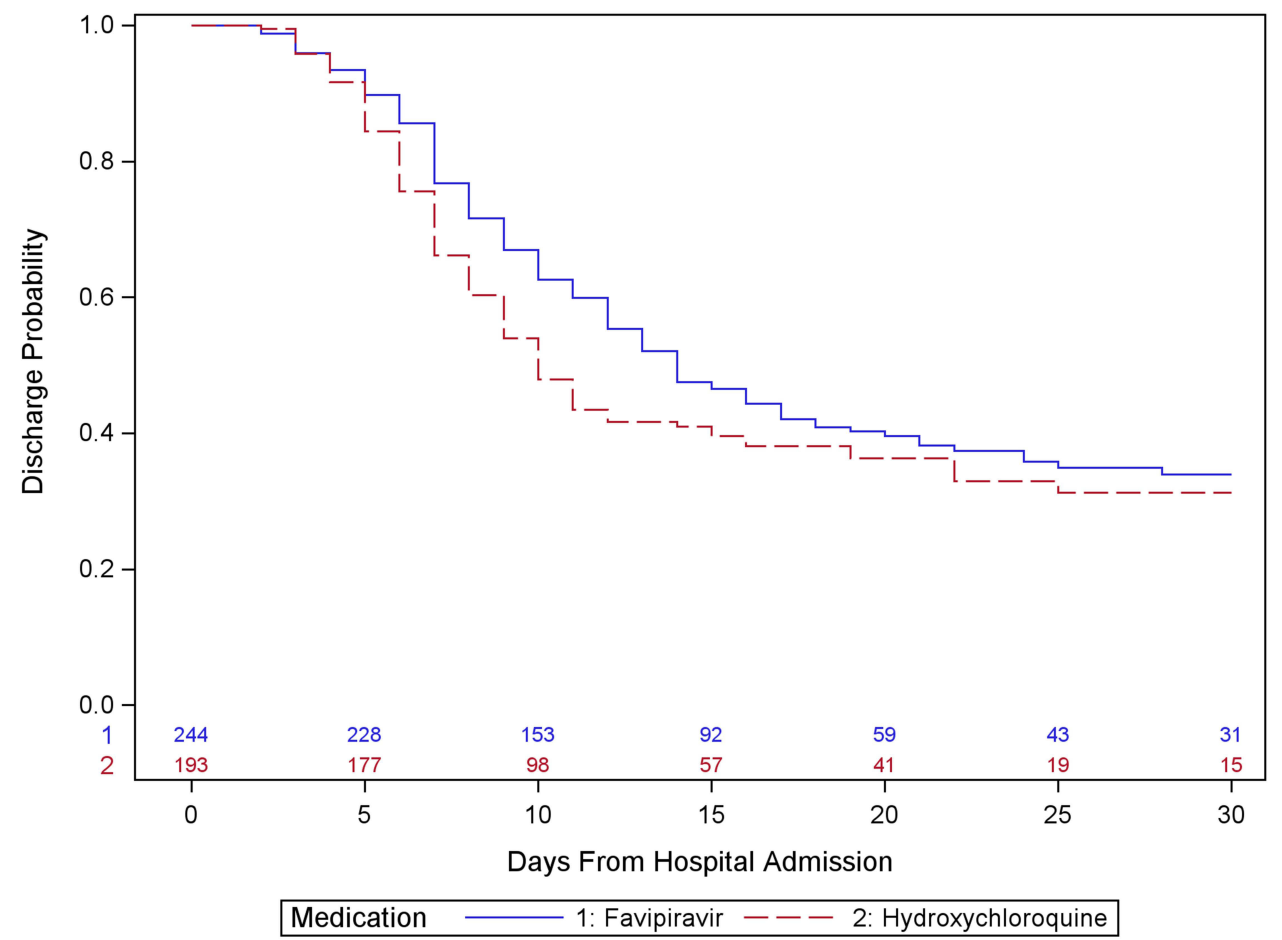

Figure 2 Kaplan-Meier discharge probability curves among two groups. 
these comorbidities around the world in COVID-19 patients. ${ }^{15,17}$ In the current study, the main symptoms and signs reported in the two groups were cough, fever, low oxygen saturation, and shortness of breath. They were reported more frequently in the HCQ than the FPV group, and the difference was significant. Similarly, a study in the Third People's Hospital of Shenzhen examined clinical results in people with COVID-19 treated with FPV and those treated with LPV-RTV. They found that patients treated with FPV recovered faster, and the symptoms and signs of both groups showed more recovery in the FPV group than in the LPV-RTV group. ${ }^{10}$

This study examined whether FPV improved COVID19 patients' clinical outcomes in terms of time to discharge and mortality compared to HCQ treatment. In the current study, the mortality rate in the FPV group (8.2\%) was higher versus patients in the HCQ group (7.3\%). Also, hospital stays of more than 14 days were significantly highest in neither medication (63.4\%) then FPV (36.9\%) and lastly HCQ (36.3\%) $(\mathrm{P}<0.0001)$. In France, Lagier et $\mathrm{al}^{18}$ reported that therapy with HCQ and azithromycin decreased hospital stay duration, risk of ICU transfer, and deaths in COVID-19 cases. However, in the present study, ICU admission with FPV group (4.3\%) compared to $(6 \%)$ HCQ group with not statistically significant $(p=0.715)$. Similarly, Guner et al compared the ICU admission rate of mild/moderate COVID-19 patients treated with HCQ, FPV, or HCQ plus FPV in the hospital setting study. They discovered no statistically significant difference in ICU admissions between the HCQ and HCQ plus FPV categories. ${ }^{19}$

In the present study, the Kaplan-Meier survival curve showed better survival analysis with the enhanced survival persisting out to 30 days from admission and improved discharge time among patients in HCQ treated group, with an insignificant difference with the FPV treated group. In addition, Udwadia et al reported that Kaplan-Meier analyses of time to clinical cure exhibited significant improvement in time to clinical cure, suggests FPV may be beneficial in mild-to-moderate COVID-19. ${ }^{20}$

In the present study, the Cox regression model for mortality prediction revealed that mechanical ventilation was predictive for mortality. Patients on mechanical ventilation had about 13 times more risk of death than others who did not receive mechanical ventilation. Numerous studies have been published in the literature that examine mortality as an outcome for patients undergoing mechanical ventilation. ${ }^{21,22}$
In the present study, the Cox regression model for discharge prediction showed that patients who prescribed neither medication had a significantly high risk of extended hospitalization after 30 days than those prescribed HCQ. In contrast, there was no significant difference between those who received FPV and those who received HCQ. Cox regression of the risk factors for discharge prediction revealed age $>65$ years and mechanical ventilation significantly high risk of extended hospitalization. According to a report conducted by Thai et $\mathrm{al}^{23}$ the average length of stay in the hospital was 21 days. The multivariable Cox regression model demonstrates that prolonged hospital stays are significantly correlated with age, residency, and source of contamination.

In the current study, the most adverse effects of FPV were increasing ALT and AST, mild to moderate diarrhea, nausea, and vomiting, as reported by others. ${ }^{24}$ In addition, the most often reported adverse events of FPV were gastrointestinal disturbances, uric acid elevations, neutrophil count decreased, ALT and AST elevations, psychological symptom reactions, and blood triglyceride elevations. ${ }^{25}$ Also, Cai et $\mathrm{al}^{10}$ reported a clinical trial in COVID-19 cases administered FPV. Adverse effects were observed in $11.4 \%$ of cases $(4 / 35)$; two had diarrhea, one had liver disorders, and one had decreased appetite. Madelain et $\mathrm{al}^{24}$ could explain that FPV is primarily metabolized in the liver by the aldehyde oxidase enzyme and metabolizes a small portion by xanthine oxidase.

Significant QT prolongation was observed in COVID19 patients treated with HCQ, a key finding in this study. However, this did not appear in patients who were given FPV. Only two small observational studies have reported associations between HCQ doses of 200$400 \mathrm{mg}$ daily and QT prolongation. ${ }^{26,27}$ Also, Çap et al reported that in the ECG recordings received in the following days after the treatment was started in COVID-19 patients, there was a significant prolongation in the QT interval with $\mathrm{HCQ}$, but there was no significant change with FPV. ${ }^{28}$ Moreover, the effect of FPV on the QT interval in healthy adults was evaluated in a small-scale study (56 participants) in Japan. Patients were randomly assigned to receive $1200 \mathrm{mg}$ FPV, $2400 \mathrm{mg} \mathrm{FPV}$, moxifloxacin, or a placebo. On ECG images obtained three and six hours after FPV administration, no significant QT interval prolongation was observed. ${ }^{29}$

Due to the retrospective nature of our study, the pandemic, and the need for a rapid response with effective treatment options, clinicians initially utilized FPV and 
HCQ without collecting some baseline data, such as C-reactive protein (CRP), D-dimer, and lymphocyte count, that may have affected treatment allocation. Moreover, the current study has other limitations in that it only included hospitalized individuals in Taif province. Therefore results may not apply to different areas in the Kingdom. In addition, only one HCQ and FPV dosing regimen were investigated; multiple doses for safety evaluation are indicated.

\section{Conclusions}

The present study concluded that FPV and HCQ showed comparable efficacy in decrease mortality and oxygen requirements. Interestingly, FPV exhibited no prolonged Q-T interval, although there was a significant comorbidities association, especially heart disease, compared to the HCQ. A big RCT is required to validate or contradict our findings.

\section{Institutional Review Board Statement}

The study was conducted according to the guidelines of the bioethics and research committee of the King Faisal Medical Complex, Taif, Saudi Arabia, and approved by Taif Research and Ethics Committee (Approval \# HAP02-T-67) under the reference number of (Reference Approval \# HAP-02-T-67).

\section{Acknowledgment}

This manuscript is a master's degree thesis that has been submitted to the Deanship of Graduate Studies, King Abdulaziz University.

\section{Disclosure}

The authors declare no conflicts of interest in this work.

\section{References}

1. Horby P, Mafham M, Linsell L, et al. Effect of hydroxychloroquine in hospitalized patients with COVID-19: preliminary results from a multi-center, randomized, controlled trial. MedRxiv. 2020. https:// www.semanticscholar.org/paper/Effect-of-Hydroxychloroquine-in$\mathrm{H}$ o s p i t a $1 \mathrm{iz}$ e d - w i t h- a - H o r b y - M a f h a m/ 9019fe8a10a26e412af464eda3a54320ed179dbf

2. Furtado RH, Berwanger O, Fonseca HA, et al. Azithromycin in addition to standard of care versus standard of care alone in the treatment of patients admitted to the hospital with severe COVID-19 in Brazil (COALITION II): a randomised clinical trial. Lancet. 2020;396 (10256):959-967. doi:10.1016/S0140-6736(20)31862-6

3. Wang Y, Zhang D, Du G, et al. Remdesivir in adults with severe COVID-19: a randomised, double-blind, placebo-controlled, multicentre trial. Lancet. 2020;395(10236):1569-1578. doi:10.1016/S01406736(20)31022-9
4. Nasir M, Perveen RA, Saha SK, Talha KA, Selina F, Islam MA. Systematic review on repurposing use of Favipiravir against SARS-CoV-2. Mymensingh Med J. 2020;29(3):747-754.

5. Lu R. Genomic characterization and epidemiology of 2019 novel coronavirus: implications for virus origins and receptor binding. Lancet. 2019:395(10224):566-568.

6. Gao J, Tian Z, Yang X. Breakthrough: chloroquine phosphate has shown apparent efficacy in treatment of COVID-19 associated pneumonia in clinical studies. Biosci Trends. 2020;14(1):72-73. doi: $10.5582 /$ bst.2020.01047

7. Wang M, Cao R, Zhang L, et al. Remdesivir and chloroquine effectively inhibit the recently emerged novel coronavirus (2019-nCoV) in vitro. Cell Res. 2020;30(3):269-271. doi:10.1038/s41422-020-0282-0

8. Commission NH. Diagnosis and treatment protocol for novel coronavirus pneumonia (Trial version 8); 2020.

9. Chen C, Huang J, Cheng Z, et al. Favipiravir versus arbidol for COVID-19: a randomized clinical trial. MedRxiv. 2020. https:// www.medrxiv.org/content/10.1101/2020.03.17.20037432v4\#: :text= Conclusions\%20Among $\% 20$ patients $\% 20$ with $\% 20$ COVID, Favipiravir\%20are\%20mild\%20and\%20manageable.

10. Cai Q, Yang M, Liu D, et al. Experimental treatment with favipiravir for COVID-19: an open-label control study. Engineering. 2020;6 (10):1192-1198. doi:10.1016/j.eng.2020.03.007

11. Saudi $\mathrm{MoH}$ protocol for patients suspected of/confirmed with COVID-19 supportive care and antiviral treatment of suspected or confirmed COVID-19 infection (version 1.4) AtS; 2020 Available from: https://www.moh.gov.sa/en/Ministry/MediaCenter/ Publications/Pages/covid19.aspx. Accessed September 3, 2021.

12. Chen J, Liu D, Liu L, et al. A pilot study of hydroxychloroquine in treatment of patients with common coronavirus disease-19 (COVID-19). J Zhejiang Univ. 2020;49(1):215-219

13. Tablet A. $200 \mathrm{mg}$. Report on the Deliberation Results. Evaluation and Licensing Division, Pharmaceutical and Food Safety Bureau. Ministry of Health. Labour and Welfare; 2014.

14. Rattanaumpawan P, Jirajariyavej S, Lerdlamyong K, Palavutitotai N, Saiyarin J. Real-world experience with favipiravir for treatment of COVID-19 in Thailand: results from a multi-center observational study. MedRxiv. 2020. https://www.medrxiv.org/content/10.1101/ 2020.06.24.20133249v3

15. Perveen RA, Nasir M, Murshed M, Nazneen R, Ahmad SN. Remdesivir and favipiravir changes hepato-renal profile in COVID-19 patients: a cross sectional observation in Bangladesh. Int J Med Sci Clin Invent. 2021;8(1):5196-5201. doi:10.18535/ijmsci/v8i01.03

16. Kaur RJ, Charan J, Dutta S, et al. Favipiravir use in COVID-19: analysis of suspected adverse drug events reported in the WHO database. Infect Drug Resist. 2020;13:4427. doi:10.2147/IDR. S287934

17. Dabbous HM, El-Sayed MH, El Assal G, et al. Safety and efficacy of favipiravir versus hydroxychloroquine in management of COVID-19: a randomised controlled trial. Sci Rep. 2021;11(1):1-7. doi:10.1038/ s41598-021-85227-0

18. Lagier J-C, Million M, Gautret P, et al. Outcomes of 3737 COVID-19 patients treated with hydroxychloroquine/azithromycin and other regimens in Marseille, France: a retrospective analysis. Travel Med Infect Dis. 2020;36:101791. doi:10.1016/j.tmaid.2020.101791

19. Guner R, Hasanoglu I, Kayaaslan B, et al. Comparing ICU admission rates of mild/moderate COVID-19 patients treated with hydroxychloroquine, favipiravir, and hydroxychloroquine plus favipiravir. $J$ Infect Public Health. 2021;14(3):365-370. doi:10.1016/j. jiph.2020.12.017

20. Udwadia ZF, Singh P, Barkate H, et al. Efficacy and safety of favipiravir, an oral RNA-dependent RNA polymerase inhibitor, in mild-to-moderate COVID-19: a randomized, comparative, open-label, multi-center, Phase 3 clinical trial. Int J Infect Dis. 2021;103:62-71. doi:10.1016/j.ijid.2020.11.142 
21. Alzeer A, BaHammam A, Masood M, Basha SJ, Alhamad E, Sohail S. Outcome of patients with severe asthma in the intensive care unit. Ann Saudi Med. 2006;26(6):461-465. doi:10.5144/02564947.2006.461

22. Mamary AJ, Kondapaneni S, Vance GB, Gaughan JP, Martin UJ, Criner GJ. Survival in patients receiving prolonged ventilation: factors that influence outcome. Clin Med Insights Circ Respir Pulm Med. 2011;5:S6649.

23. Thai PQ, Son DT, Van HTH, et al. Factors associated with the duration of hospitalisation among COVID-19 patients in Vietnam: a survival analysis. Epidemiol Infect. 2020;148. https://pubmed.ncbi. nlm.nih.gov/32517822/

24. Madelain V, Nguyen THT, Olivo A, et al. Ebola virus infection: review of the pharmacokinetic and pharmacodynamic properties of drugs considered for testing in human efficacy trials. Clin Pharmacokinet. 2016;55(8):907-923. doi:10.1007/s40262-0150364-1

25. Joshi S, Parkar J, Ansari A, et al. Role of favipiravir in the treatment of COVID-19. Int J Infect Dis. 2020;102:501-508. doi:10.1016/j. ijid.2020.10.069
26. Chen C-Y, Wang F-L, Lin -C-C. Chronic hydroxychloroquine use associated with QT prolongation and refractory ventricular arrhythmia. Clinical Toxicol. 2006;44(2):173-175.

27. Negoescu A, Thornback A, Wong E, Ostor A. Long QT and hydroxychloroquine; A poorly recognised problem in rheumatology patients.: 2045. Arthritis Rheum. 2013;65. https://acrabstracts.org/ abstract/long-qt-and-hydroxychloroquine-a-poorly-recognised-pro blem-in-rheumatology-patients/

28. Çap M, Bilge Ö, Işı F, et al. The effect of favipiravir on QTc interval in patients hospitalized with coronavirus disease 2019. $J \quad$ Electrocardiol. 2020;63:115-119. doi:10.1016/j.jelectroc ard.2020.10.015

29. Kumagai Y, Murakawa Y, Hasunuma T, et al. Lack of effect of favipiravir, a novel antiviral agent, on QT interval in healthy Japanese adults. Int J Clin Pharmacol Ther. 2015;53(10):866-874. doi: $10.5414 / \mathrm{CP} 202388$

\section{Publish your work in this journal}

The International Journal of General Medicine is an international, peer-reviewed open-access journal that focuses on general and internal medicine, pathogenesis, epidemiology, diagnosis, monitoring and treatment protocols. The journal is characterized by the rapid reporting of reviews, original research and clinical studies across all disease areas. The manuscript management system is completely online and includes a very quick and fair peer-review system, which is all easy to use. Visit http://www.dovepress.com testimonials.php to read real quotes from published authors. 